\title{
Metastable configurations of spin models on random graphs
}

\author{
Johannes Berg and Mauro Sellitto \\ Abdus Salam International Centre for Theoretical Physics, 34100 Trieste, Italy
}

\begin{abstract}
One-flip stable configurations of an Ising-model on a random graph with fluctuating connectivity are examined. In order to perform the quenched average of the number of stable configurations we introduce a global order-parameter function with two arguments. The analytical results are compared with numerical simulations.
\end{abstract}

\section{INTRODUCTION}

Spin-models on random graphs have a long history in the statistical mechanics of disordered systems. A random graph [1] consists of $N$ nodes where each node is linked at random to a finite number of other nodes. The resulting structure is locally tree-like but has loops of a length $\ln (N)$. Associating each node $i$ with an Ising-spin variable $s_{i}= \pm 1$ and each connection with a bond $J_{i j}$ - giving a contribution $-J_{i j} s_{i} s_{j}$ to the Hamiltonian - defines a spin-model on a random graph. Loops in the graph are the source of frustration of the model.

As a model of spin glasses random-graph models are particularly attractive [2], since they combine the analytic accessibility within the framework of mean-field theory [3 5 with the finite connectivity of short-range, finite-dimensional models. Furthermore, random-graph models occur in problems of combinatorial optimisation and theoretical computer science [6]-7], where solving e.g. a satisfiability or a matching problem is equivalent to finding the ground-state of a spin-model on a graph. Considering statistical ensembles of such problems with the aim of characterizing typical problems corresponds to defining an ensemble of random graphs. Interest in random-graph models has intensified lately and led to solution schemes beyond replica-symmetry [8 11].

For fully connected systems, where the local field at each site is the sum of many random terms, the central limit theorem ensures that the local fields are Gaussian distributed. The distribution of fields may thus be characterized at the level of replica symmetry - by two variables, the mean and the variance of this distribution. The free energy is thus characterized by a few order-parameters, which are determined self-consistently. Finite-connectivity models on random graphs are mean-field models where the local fields do not consist of many terms and consequently are not Gaussian distributed. The free energy is characterized by a continuous order-parameter function - the distribution of local fields. A key step simplifying the replica analysis of random graphs models has been the introduction of a global order-parameter function [8.9]. In the case of Hamiltonians containing 2-spin, 3-spin, and higher interaction terms, the sums emerging from averaging over the disorder may be disentangled using the order parameter function $c(\underline{\sigma})=1 / N \sum_{i} \delta_{\underline{\sigma} \underline{s}_{i}}$. This function gives the fraction of sites, whose replicated spins $\underline{s}_{i}=\left\{s_{i}^{a}\right\}(a=1 \ldots n)$ are in a given configuration $\underline{\sigma}$.

In principle any function of the spin configuration may be written as a sum of 2-spin, 3 -spin, and higher interaction terms and used as an energy function. However correlations between these interactions may make the resulting Hamiltonian intractable. In this paper we discuss how to treat random-graph models with Hamiltonians with a non-trivial dependence on the local magnetic field at each site.

The central problem in this case is to find an order-parameter function to disentangle the result of averaging terms of the form $\exp \left\{i \sum_{a} s_{i}^{a} J_{i j} \hat{h}_{j}^{a}\right\}$ over the disorder $J_{i j}$ (instead of the simpler $\exp \left\{i \sum_{a} s_{i}^{a} J_{i j} s_{j}^{a}\right\}$, the variables $\hat{h}_{i}^{a}$ emerge from defining the local fields $h_{i}^{a}$ ). Furthermore such an order-parameter function ought to admit a replica-symmetric ansatz or other schemes and an analytic continuation $n \rightarrow 0$. In this paper we show that this need is answered by a two-argument order-parameter function $c(\underline{\sigma}, \underline{\tau})=1 / N \sum_{i} \delta_{\underline{\underline{\sigma}} \underline{\underline{s}}_{i}} \mathrm{e}^{\hat{\mathrm{h}}_{i}} \cdot \underline{\underline{\tau}}$. Viewing the variables $\hat{h}_{i}^{a}, h_{i}^{a}$ as new phase-space variables coupled to the spins, this order parameter function may be viewed as a discrete Fourier-transform of the generalisation of the usual order-parameter function $c(\underline{\sigma})$ to $c(\underline{\sigma}, \underline{\hat{h}})$ (the local fields may be integrated out explicitly).

As a concrete example we calculate the quenched average in replica-symmetry of the number of metastable configurations in one of the simplest model on a random graph, the ferromagnetic 2-spin model. For this model some results have already been obtained, albeit restricted to the annealed approximation, both for graphs with fixed connectivity 12] and fluctuating connectivity [13]. For fully-connected disordered systems, like the Sherrington-Kirkpatrick model, the problem of metastable states has been dealt with in the classic paper by Bray and Moore 14. Metastable configurations have each spin pointing in the direction of its local field, i.e. they are stable (or marginally stable) against single-spin flips. As so-called inherent structures, such configurations play a crucial role in structural glasses [15, 16. In spin-models of dense granular matter they play the role of blocked configurations 17 19. Hamiltonians which non-trivially depend on the local magnetisation also occur in the context of lattice-gases, e.g. where sites with more than a certain number of neighbouring particles are energetically penalized [20]. Finally, a treatment of the 
dynamics of spin models on random graphs will need an order-parameter function (generating function) analogous to the one introduced here, since the dynamics depends explicitly on the local field at each point.

The paper is organized as follows: First the ferromagnetic 2-spin model on a random graph is introduced. After discussing the annealed approximation to the problem, the calculation of the quenched average of the number of metastable configurations is outlined in section II. Particular emphasis is given to the 2-argument global orderparameter function. The replica-symmetric ansatz for the order-parameter function is discussed and the replicasymmetric result for the entropy of metastable configurations is given. These expressions are evaluated numerically and are compared to the results of Monte-Carlo simulations and thermodynamic integration of an auxiliary model in section [II. The results of generalizing the problem to 3-spin models are given in section IV] and the generalisation to models with frustrated interactions is discussed in section $\mathrm{V}$.

\section{METASTABLE CONFIGURATIONS OF THE 2-SPIN FERROMAGNETIC MODEL}

In the following we consider one of the simplest spin-models on a random graph, namely the two-spin ferromagnetic model defined by the Hamiltonian

$$
H=-\sum_{i<j} C_{i j} s_{i} s_{j}
$$

where the sites $i=1, \ldots, N$ and $s_{i}= \pm 1$. The variable $C_{i j}=1$ with $i<j$ denotes the presence of a bond connecting sites $i, j$ and $C_{i j}=0$ denotes its absence. Choosing $C_{i j}=1(0)$ randomly with probability $c / N(1-c / N)$ defines an ensemble of random graphs, where the number of bonds connected to a site is distributed with a Poisson distribution of finite average $c$.

The condition for a (marginally) metastable configuration is that at each site $i$ the local field $h_{i}=\sum_{j} C_{i j} s_{j}$ obeys $h_{i} s_{i} \geq 0$. This definition implies that a number of neutral moves remains, as spins with zero local field may be flipped without a cost in energy. We choose this definition, since a quench of the system will in general leave a number of spins with zero local field, which are crucial to the subsequent dynamics [21]. Nevertheless the marginally stable states could be excluded easily by considering only configurations with non-zero local magnetic field.

\section{A. The annealed approximation}

It is instructive to see how the average number of blocked configurations is calculated in the annealed approximation, where the partition function is averaged directly over the ensemble of random graphs, before passing to the more complicated case of the quenched average.

The partition function configurations $Z(\beta)$ may be written as

$$
Z(\beta)=\prod_{i}\left(\sum_{s_{i}= \pm 1} \sum_{h_{i}=-\infty}^{\infty} \delta\left(h_{i} ; \sum_{j} C_{i j} s_{j}\right) \Theta\left(h_{i} s_{i}\right)\right) \exp \left\{\beta / 2 \sum_{i} h_{i} s_{i}\right\}
$$

where $\delta(x ; y)=1$ if $x=y$ and 0 otherwise, denotes a Kronecker-delta and $\Theta(x)$ denotes a discrete Heaviside stepfunction with $\Theta(x)=1$ if $x \geq 0$ and 0 otherwise. The function $\prod_{i} \Theta\left(h_{i} s_{i}\right)$ denotes the condition for a metastable configuration. However none of the subsequent steps of the calculation affect this function and it may be used to encode any function of $h_{i}$ and $s_{i}$. The average over the ensemble of random graphs with connectivity $c$ may be written as

$$
\langle\langle(.)\rangle\rangle=\prod_{i<j} \int d C_{i j}\left[(1-c / N) \delta\left(C_{i j}\right)+c / N \delta\left(C_{i j}-1\right)\right](.)
$$

We use the integral representation $\delta\left(h_{i} ; \sum_{j} C_{i j} s_{j}\right)=\int_{0}^{2 \pi} d \hat{h}_{i} \exp \left\{-i h_{i} \hat{h}_{i}+i \sum_{j} \hat{h}_{i} C_{i j} s_{j}\right\}$ for the Kronecker-deltas, so the average over the disorder yields a term of the form

$$
\prod_{i<j}\left[1-c / N+c / N \exp \left\{i \hat{h}_{i} s_{j}+i \hat{h}_{j} s_{i}\right\}\right]=\lim _{N \rightarrow \infty} \exp \left\{-c N / 2+c /(2 N) \sum_{i, j} \mathrm{e}^{i \hat{h}_{i} s_{j}+i \hat{h}_{j} s_{i}}\right\} \text {. }
$$


The sum over the site labels $i$ and $j$ may be disentangled by introducing $c_{\sigma}^{\tau}=1 / N \sum_{i} \delta_{s_{i} \sigma} e^{i \hat{h}_{i} \tau}$ for $\sigma, \tau= \pm 1$. Using these 4 order parameters, the average over the disorder may be written as

$$
\exp \left\{-c N / 2+c /(2 N) \sum_{i, j} \mathrm{e}^{i \hat{h}_{i} s_{j}+i \hat{h}_{j} s_{i}}\right\}=\exp \left\{-c N / 2+c N / 2 \sum_{\sigma, \tau} c_{\sigma}^{\tau} c_{\tau}^{\sigma}\right\},
$$

where the symmetry of the exponent in (4) under interchange of the site labels $i$ and $j$ is reflected by the symmetry of the exponent of (5) under exchange of $\sigma$ and $\tau$.

After standard manipulations, one now easily obtains the annealed average of the free energy

$$
\begin{aligned}
& 1 / N \ln \langle\langle Z(\beta)\rangle\rangle=\operatorname{extr}_{c_{ \pm}^{ \pm}}\left[-c / 2\left(c_{+}^{+2}+2 c_{-}^{+} c_{+}^{-}+c_{-}^{-2}\right)-c / 2\right. \\
& \left.+\ln \left[\sum_{h=1}^{\infty}\left(e^{\beta / 2} \sqrt{c_{-}^{-} / c_{+}^{-}}\right)^{h} I_{h}\left(2 c \sqrt{c_{-}^{-} c_{+}^{-}}\right)+I_{0}\left(2 c \sqrt{c_{-}^{-} c_{+}^{-}}\right)+I_{0}\left(2 c \sqrt{c_{+}^{+} c_{-}^{+}}\right)+\sum_{h=1}^{\infty}\left(e^{\beta / 2} \sqrt{c_{+}^{+} / c_{-}^{+}}\right)^{h} I_{h}\left(2 c \sqrt{c_{+}^{+} c_{-}^{+}}\right),\right]\right]
\end{aligned}
$$

where $I_{h}(x)$ denotes the modified Bessel-function of the first kind of order $h$. The extremum is over the order parameters $c_{\sigma}^{\tau}$.

\section{B. The quenched average}

Using the replica trick $\ln Z=\lim _{n \rightarrow 0} \partial_{n} Z^{n}$ to represent the logarithm of the partition function, the quenched average over the ensemble of random graphs of the free energy of metastable configurations may be written as

$$
\begin{aligned}
& \left\langle\left\langle Z^{n}(\beta)\right\rangle\right\rangle=\prod_{i<j} \int d C_{i j}\left[(1-c / N) \delta\left(C_{i j}\right)+c / N \delta\left(C_{i j}-1\right)\right] \\
& \prod_{i, a}\left(\sum_{s_{i}^{a}= \pm 1} \sum_{h_{i}^{a}=-\infty}^{\infty} \delta\left(h_{i}^{a} ; \sum_{j} C_{i j} s_{j}^{a}\right) \Theta\left(h_{i}^{a} s_{i}^{a}\right)\right) \exp \left\{\beta / 2 \sum_{i, a} h_{i}^{a} s_{i}^{a}\right\},
\end{aligned}
$$

where the sum over the site indices $i$ is from 1 to $N$ and the sum over the replica indices goes from 1 to $n$, where $n$ is taken to be an integer. The Kronecker-deltas defining $h_{i}^{a}$ are again represented using auxiliary integrals over the auxiliary variables $\hat{h}_{i}^{a}$ giving

$$
\begin{aligned}
& \left\langle\left\langle Z^{n}(\beta)\right\rangle\right\rangle=\prod_{i<j} \int d C_{i j}\left[(1-c / N) \delta\left(C_{i j}\right)+c / N \delta\left(C_{i j}-1\right)\right] \\
& \prod_{i, a}\left(\sum_{s_{i}^{a}= \pm 1} \sum_{h_{i}^{a}=-\infty}^{\infty} \int_{0}^{2 \pi} d \hat{h}_{i}^{a} /(2 \pi)\right) \exp \left\{-i \sum_{i, a} h_{i}^{a} \hat{h}_{i}^{a}+i \sum_{i, j, a} \hat{h}_{i}^{a} C_{i j} s_{j}^{a}+\beta / 2 \sum_{i, a} h_{i}^{a} s_{i}^{a}\right\} \prod_{i, a} \Theta\left(h_{i}^{a} s_{i}^{a}\right),
\end{aligned}
$$

so that the disorder-term $C_{i j}$ occurs only a single time in the exponent. The average over the term containing the disorder thus yields

$$
\prod_{i<j}\left[1-c / N+c / N \exp \left\{i \sum_{a} \hat{h}_{i}^{a} s_{j}^{a}+i \sum_{a} \hat{h}_{j}^{a} s_{i}^{a}\right\}\right]=\lim _{N \rightarrow \infty} \exp \left\{-c N / 2+c /(2 N) \sum_{i, j} \mathrm{e}^{i \sum_{a} \hat{h}_{i}^{a} s_{j}^{a}+i \sum_{a} \hat{h}_{j}^{a} s_{i}^{a}}\right\} .
$$

The crucial problem at this point is to find an order-parameter function suitable to decouple the sums over the sites in the exponent. A natural choice would be $1 / N \sum_{i} \delta\left(\underline{\hat{h}}-\underline{\hat{h}_{i}}\right) \delta_{\underline{\sigma} \underline{s}_{i}}$ where the underlined terms are used to denote vectors in replica space, i.e. $\underline{\sigma}=\left\{\sigma^{a}\right\}$. However $\underline{\hat{h}}$ is a vector of continuous variables, which means that there is no simple replica-symmetric ansatz for this order-parameter function. Instead we generalize the order parameters used in the annealed approximation in section $\amalg \mathrm{IA}$ and define $c(\underline{\sigma}, \underline{\tau})=1 / N \sum_{i} \delta_{\underline{\sigma} \underline{s}_{i}} \mathrm{e}_{\underline{\underline{h}}} \cdot \underline{\tau}$, where $\underline{\tau}$ is another binary vector in replica space. Using this global order-parameter function of two arguments (9) becomes

$$
\exp \left\{-c N / 2+c N / 2 \sum_{\underline{\sigma}, \underline{\underline{\tau}}} c(\underline{\sigma}, \underline{\tau}) c(\underline{\tau}, \underline{\sigma})\right\} .
$$


After some formal manipulations outlined in the appendix one obtains as the replicated partition-function

$$
\begin{aligned}
& \left\langle\left\langle Z^{n}(\beta)\right\rangle\right\rangle=\prod_{\underline{\sigma}, \underline{\tau}} \int d c(\underline{\sigma}, \underline{\tau}) \exp \left\{-c N / 2 \sum_{\underline{\sigma}, \underline{\underline{\tau}}} c(\underline{\sigma}, \underline{\tau}) c(\underline{\tau}, \underline{\sigma})-c N / 2\right\} \\
& {\left[\sum_{\underline{\sigma}, \underline{h}} \int_{0}^{2 \pi} d \underline{\hat{h}} /(2 \pi)^{n} \exp \left\{-i \underline{h} \cdot \underline{\hat{h}}+1 / 2 \beta \underline{h} \cdot \underline{\sigma}+c \sum_{\underline{\tau}} c(\underline{\tau}, \underline{\sigma}) \mathrm{e}^{i \hat{\hat{h}} \cdot \underline{\underline{\tau}}\}} \prod_{a} \Theta\left(h^{a} \sigma^{a}\right)\right]^{N} .\right.}
\end{aligned}
$$

The integral over the order-parameter function may be performed in the thermodynamic limit $N \rightarrow \infty$ as a saddlepoint integral. Using the shorthand $\mathrm{e}^{\Lambda}$ for the term in square brackets in (11) we obtain the self-consistent equation for the order-parameter function

$$
c(\underline{\sigma}, \underline{\tau})=\mathrm{e}^{-\Lambda} \sum_{k=0}^{\infty} \frac{c^{k}}{k !} \prod_{l=1}^{k}\left(\sum_{\underline{\rho}_{l}} c\left(\underline{\rho}_{l}, \underline{\sigma}\right)\right) \exp \left\{1 / 2 \beta \sum_{a}\left(\tau^{a}+\sum_{l} \rho_{l}^{a}\right) \sigma^{a}\right\} \prod_{a} \Theta\left(\sigma^{a}\left(\tau^{a}+\sum_{l} \rho_{l}^{a}\right)\right),
$$

where $\rho_{l}^{a}$ is summed over the values \pm 1 . The assumption that $c(\underline{\sigma}, \underline{\tau})$ is real-valued is consistent with this equation.

To treat this equation in the limit $n \rightarrow 0$ we need to make an ansatz concerning the form of the order-parameter function. The simplest possible choice is the replica-symmetric (RS) ansatz, which assumes that the order-parameter function is invariant under the permutation of the replica-indices [22]. This in turn implies that $c(\underline{\sigma}, \underline{\tau})$ is a function of $\sum_{a} \sigma^{a}, \sum_{a} \sigma^{a} \tau^{a}$, and $\sum_{a} \tau^{a}$ only. The replica-symmetric order-parameter function may thus be written as

$$
c(\underline{\sigma}, \underline{\tau})=\int d x d y d z P(x, y, z) \frac{\exp \left\{\beta x \sum_{a} \sigma^{a}+\beta y \sum_{a} \sigma^{a} \tau^{a}+\beta z \sum_{a} \tau^{a}\right\}}{\left[2 \mathrm{e}^{\beta y} \cosh (\beta(x+z))+2 \mathrm{e}^{-\beta y} \cosh (\beta(x-z))\right]^{n}},
$$

where the denominator serves to normalize $\int d x d y d z P(x, y, z)=\sum_{\underline{\sigma}, \underline{\tau}} c(\underline{\sigma}, \underline{\tau})$.

Inserting the RS-ansatz (13) into the self-consistent equation (12) and taking the limit $n \rightarrow 0$ one finally obtains the self-consistent equation for $P(x, y, z)$ in the form of an invariant density

$$
\begin{aligned}
P(x, y, z)=\mathrm{e}^{-c} \sum_{k=0}^{\infty} \frac{c^{k}}{k !} & \prod_{l=1}^{k} \int d x_{l} d y_{l} d z_{l} P\left(x_{l}, y_{l}, z_{l}\right) \delta\left(x-\frac{1}{4 \beta} \ln \left(\frac{f_{++} f_{+-}}{f_{-+} f_{--}}\right)\right) \\
& \delta\left(y-\frac{1}{4 \beta} \ln \left(\frac{f_{++} f_{--}}{f_{-+} f_{+-}}\right)\right) \delta\left(z-\frac{1}{4 \beta} \ln \left(\frac{f_{++} f_{-+}}{f_{+-} f_{--}}\right)\right),
\end{aligned}
$$

where $f_{\sigma \tau}$ serves as a shorthand for

$$
f_{\sigma \tau}=f\left(\left\{x_{l}, y_{l}, z_{l}\right\}, \sigma, \tau\right)=\prod_{l=1}^{k} \sum_{\rho_{l}} \exp \left\{\beta \sum_{l} x_{l} \rho_{l}+\beta \sum_{l} y_{l} \rho_{l} \sigma+\beta \sum_{l} z_{l} \sigma+\beta / 2 \sigma \sum_{l} \rho_{l}+\beta / 2 \sigma \tau\right\} \Theta\left(\sigma\left(\tau+\sum_{l} \rho_{l}\right)\right) \text {. }
$$

Without the last term - which encodes the blocking condition - this expression would factorize in $l$.

From the RS order-parameter function $P(x, y, z)$ at a given value of $\beta$ one may also derive the values of physical observables, such as the energy,

$$
\begin{aligned}
& \left\langle\left\langle\left\langle-\frac{1}{2 N} \sum_{i} s_{i} h_{i}\right\rangle\right\rangle\right\rangle=\mathrm{e}^{-c} \sum_{k=0}^{\infty} \frac{c^{k}}{k !} \prod_{l=1}^{k} \int d x_{l} d y_{l} d z_{l} P\left(x_{l}, y_{l}, z_{l}\right) \\
& \frac{\sum_{\sigma} \prod_{l}^{k} \sum_{\rho_{l}}\left(-1 / 2 \sigma \sum_{l} \rho_{l}\right) \exp \left\{\beta \sum_{l} x_{l} \rho_{l}+\beta \sum_{l} y_{l} \rho_{l} \sigma+\beta \sum_{l} z_{l} \sigma+\beta / 2 \sigma \sum_{l} \rho_{l}\right\} \Theta\left(\sigma \sum_{l} \rho_{l}\right)}{\sum_{\sigma} \prod_{l}^{k} \sum_{\rho_{l}}},
\end{aligned}
$$

or the fraction of sites with a given local field $h$

$$
\begin{aligned}
& \left\langle\left\langle\left\langle-\frac{1}{2 N} \sum_{i} \delta_{h ; h_{i}}\right\rangle\right\rangle\right\rangle=\mathrm{e}^{-c} \sum_{k=0}^{\infty} \frac{c^{k}}{k !} \prod_{l=1}^{k} \int d x_{l} d y_{l} d z_{l} P\left(x_{l}, y_{l}, z_{l}\right)
\end{aligned}
$$

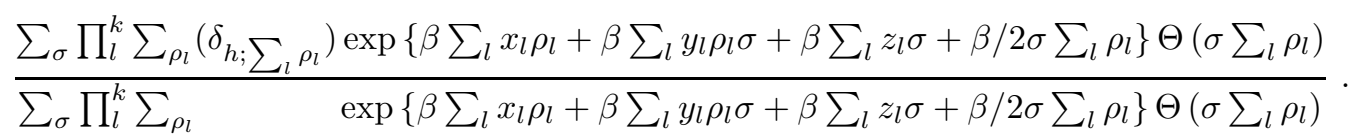


The single pointed brackets \langle\rangle serve as a shorthand for the average over the metastable configurations as defined by the partition-function (8).

Finally, inserting the RS-ansatz (13) into the partition-function (11), the free energy of blocked configurations averaged over the disorder may be obtained

$$
\begin{aligned}
\frac{1}{N}\langle\langle\ln Z\rangle\rangle= & -\beta f(\beta)=-c / 2 \prod_{l=1}^{2} \int d x_{l} d y_{l} d z_{l} P\left(x_{l}, y_{l}, z_{l}\right) \\
& \ln \left[\frac{\mathrm{e}^{\beta\left(y_{1}+y_{2}\right)} \cosh \left(\beta\left(x_{1}+z_{1}+x_{2}+z_{2}\right)\right)+\mathrm{e}^{-\beta\left(y_{1}+y_{2}\right)} \cosh \left(\beta\left(x_{1}-z_{1}-x_{2}+z_{2}\right)\right)}{2 \prod_{l=1}^{2}\left(\mathrm{e}^{\beta y_{l}} \cosh \left(\beta\left(x_{l}+z_{l}\right)\right)+\mathrm{e}^{-\beta y_{l}} \cosh \left(\beta\left(x_{l}-z_{l}\right)\right)\right)}\right] \\
& +\mathrm{e}^{-c} \sum_{k=0}^{\infty} \frac{c^{k}}{k !} \prod_{l=1}^{k} \int d x_{l} d y_{l} d z_{l} P\left(x_{l}, y_{l}, z_{l}\right) \\
& \ln \left[\frac{\sum_{\sigma} \prod_{l}^{k} \sum_{\rho_{l}} \exp \left\{\beta \sum_{l} x_{l} \rho_{l}+\beta \sum_{l} y_{l} \rho_{l} \sigma+\beta \sum_{l} z_{l} \sigma+\beta / 2 \sigma \sum_{l} \rho_{l}\right\} \Theta\left(\sigma \sum_{l} \rho_{l}\right)}{\prod_{l}^{k}\left(2 \mathrm{e}^{\beta y_{l}} \cosh \left(\beta\left(x_{l}+z_{l}\right)\right)+2 \mathrm{e}^{-\beta y_{l}} \cosh \left(\beta\left(x_{l}-z_{l}\right)\right)\right)}\right] .
\end{aligned}
$$

Details of the calculations leading to these expressions may be found in the appendix.

\section{Evaluation of the RS order-parameter function}

Since the order-parameter function $P(x, z, y)$ depends on three variables, the solution of the self-consistent equation (14) and the evaluation of (16)-(18) pose a formidable challenge. However the form of (14) as an invariant density suggests the use of a simple population dynamics to solve the self-consistent equation and evaluate the entropy of metastable configurations and other physical quantities. Recently this population dynamics has been used extensively in [10]. For the present problem it may be adopted as follows: We consider a large number $\mathcal{N}$ of triples labeled $i=1, \ldots, \mathcal{N}$ of numbers $\left\{x_{i}, y_{i}, z_{i}\right\}$ with $P(x, y, z)=1 / \mathcal{N} \sum_{i} \delta\left(x-x_{i}\right) \delta\left(y-y_{i}\right) \delta\left(z-z_{i}\right)$. The self-consistent equation (14) may then be solved numerically according to the following scheme:

- choose an integer $k$ at random according to the Poisson distribution with mean $c$.

- choose $k$ triples at random and use them to compute $f_{--}, f_{-+}, f_{+-}, f_{++}$according to (15).

- chose another triple $i$ at random and set $x_{i}=\frac{1}{4 \beta} \ln \left(\frac{f_{++} f_{+-}}{f_{-+} f_{--}}\right), y_{i}=\frac{1}{4 \beta} \ln \left(\frac{f_{++} f_{--}}{f_{-+} f_{+-}}\right), z_{i}=\frac{1}{4 \beta} \ln \left(\frac{f_{++} f_{-+}}{f_{+-} f_{--}}\right)$.

Repeating these steps a sufficiently large number of times to ensure convergence yields an approximation for the order-parameter function $P(x, z, y)$, whose quality depends on the number of triples $\mathcal{N}$ used.

In the same way the expressions (16)-(18) may be evaluated. Choosing a set of $k$ triples at random (where $k$ is sampled from the appropriate distribution) the integrands of $(16)-(18)$ may be computed. Averaging the results of sufficiently many such steps we obtain approximations of the multiple integrals over $x, y, z$ in these expressions.

The results discussed in the following section were obtained with $\mathcal{N}=5000,350000$ iterations steps, and 50000 steps to calculate (16)-(18).

\section{Numerical simulation}

To check our analytical results - evaluated numerically - against the results of numerical simulations, we use a method based on Monte-Carlo simulation of an auxiliary model and thermodynamic integration. Denoting the number of sites with $h_{i} s_{i} \geq 0$ as $N_{b}$ we define the auxiliary Hamiltonian $\mathcal{H}$

$$
\beta_{\text {aux }} \mathcal{H}=\beta H+\beta_{\operatorname{aux}}\left(N-N_{b}\right),
$$

where $H$ is the original Hamiltonian (11) and $\beta$ is a Lagrange multiplier fixing the energy $E$ of the original system. By construction, the states accessed in the limit $\beta_{\mathrm{aux}} \rightarrow \infty$ of $\mathcal{H}$ are the blocked configuration of the original Hamiltonian with energy $E$ fixed by the parameter $\beta$ (provided they exist) [23]. Numerically, blocked configurations of (1) of a given energy may thus be generated by a Monte-Carlo dynamics of the auxiliary Hamiltonian (19), starting at a low auxiliary temperature $1 / \beta_{\text {aux }}$, and gradually increasing $\beta_{\text {aux }}$ until the ground state is found. The auxiliary temperature must be changed sufficiently gradually to ensure the system always remains in equilibrium. 
Having taken the limit $\beta_{\text {aux }} \rightarrow \infty$ the Lagrange multiplier $\beta$ is simply the inverse temperature of the blocked configurations. Thus the entropy of the blocked configurations may be obtained up to a constant by thermodynamic integration

$$
\int_{E(\beta=\infty)}^{E(\beta)} d E \beta=S(\beta)-S(\beta=\infty) .
$$

The numerical results of the following section were generated by annealing (19) on four realisations of a random graph with $N=1024$ increasing $\beta$ aux from 0 to 10 in steps 0.001 every 2000 Monte-Carlo sweeps. At the end of this process the energy $E$, the fraction of sites with zero local field, and the magnetisation were measured during the last 250 Monte-Carlo sweeps. Furthermore we checked the independence of the results on the annealing rate. In the case of the entropy, the constant of integration was obtained simply by fitting the resulting curve to the analytic result.

\section{RESULTS FOR THE 2-SPIN MODEL}

In the following we describe the results of the calculations of the preceding section for the case $c=2$ and compare them to the results of numerical simulations. In figure 1 we plot both the annealed and the quenched result for the entropy of blocked configurations. The results of the quenched average evaluated according to the algorithm of section II fluctuate somewhat, so the curve is not very smooth, especially at negative temperatures. Nevertheless, very good agreement between the results of numerical simulation according to section II D and the quenched average of the entropy is found. The maximum of the entropy is reached at energies around -.57 , so a randomly chosen blocked configuration will have this energy with a probability approaching 1 in the thermodynamic limit. In the ground state $E=-1$, all spins of a cluster of connected points are aligned. Hence the entropy density of blocked configurations is simply the number of disconnected clusters of the graph times $\log (2)$. Using the standard results of random graph theory [1] we obtain a ground state entropy of $0.1122226 \ldots$, which agrees with the present result to within numerical precision.

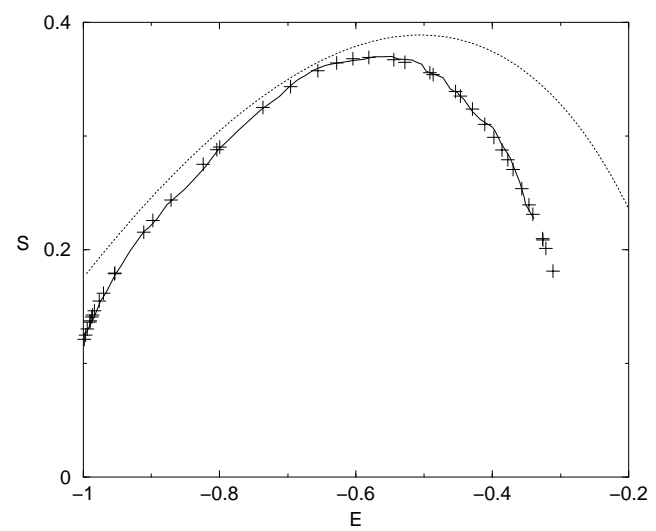

FIG. 1. The entropy density of metastable configurations as a function of their energy density $E$. The quenched result is given by the solid line, for comparison we also give the result of the corresponding annealed calculation from section IIA. Plus-signs $(+)$ denote the results of numerical simulations. At high energies (large negative temperatures) numerical problems with the algorithm used to solve the saddle-point equations arise.

In figure 2 we show the fraction of sites with zero local magnetic field as a function of the energy density $E$, which increases monotonously with $E$. This effect arises since blocked sites with non-zero magnetic field give a negative contribution to the energy. In order to obtain blocked states also at high energies, the system must thus make more of the local fields equal to zero. This is also the reason for the decrease of the entropy at increasing energies. 


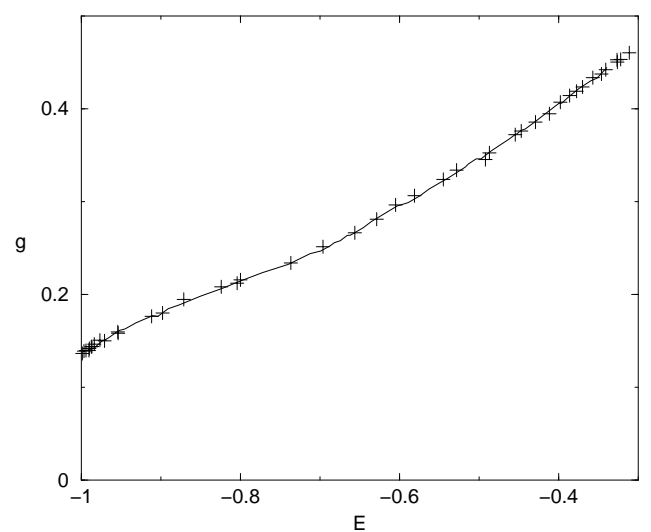

FIG. 2. The fraction of sites $g$ with zero local magnetic field as a function of the energy density $E$. Plus-signs (+) denote the results of numerical simulations.

In figure 3 we plot the absolute value of the magnetisation of blocked configurations against the energy density. There is a second-order phase-transition from configurations with zero magnetic field at high energies, to a ferromagnetic phase at low energies. Again the mechanism for this is simple: in order to form blocked configurations at low energies, (absolutely) large local fields are required. These are achieved most easily by giving the system a finite magnetisation. This simply reflects the analogous transition in the model without the blocking condition, although the transition occurs at a lower energy. At the transition, the RS order-parameter function changes qualitatively: in the high-temperature phase, its weight is concentrated at $x=z=0$ and it remains a non-trivial function only of $y$, i.e. $P(x, y, z)=\delta(x) f(y) \delta(z)$, whereas in the low-temperature phase $P(x, y, z)$ is a non-trivial function of all its arguments.

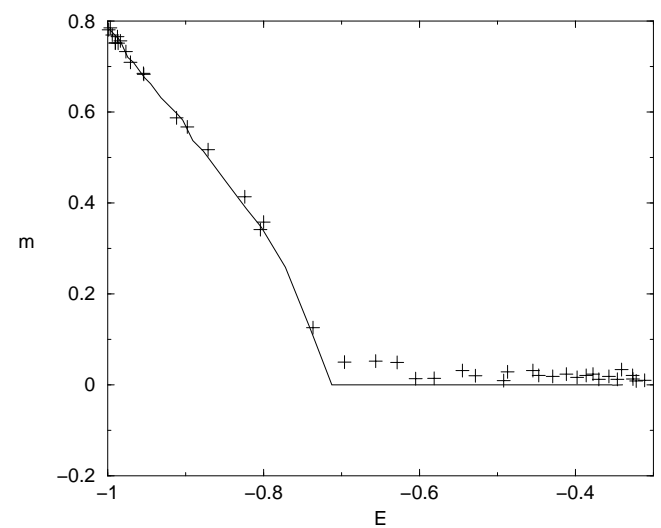

FIG. 3. The absolute value of the magnetisation as a function of the energy density E. Plus-signs $(+)$ denote the results of numerical simulations.

In figure 1 we plot the energy versus the inverse temperature of the blocked configurations $\beta$ for three connectivities $c=2,2.5,3$. We note that the kink in the curve - signifying the second-order nature of the phase transition becomes more pronounced at higher connectivities. At $c=3$ it appears that there is a jump in the curve, which would signify that the transition had become first order. Furthermore, for finite running times, the algorithm solving the saddle-point equations shows hysteresis. However none of this is borne out by closer analysis. Near the transition, the three curves in figure 4 collapse onto each other when rescaled by the width $\delta \beta$ over which the transition occurs. Furthermore the hysteresis in the algorithm disappears when a small magnetic field is applied. We thus conclude that at high connectivities, the transition remains of second order, but is characterized by a long plateau at the transition point. 


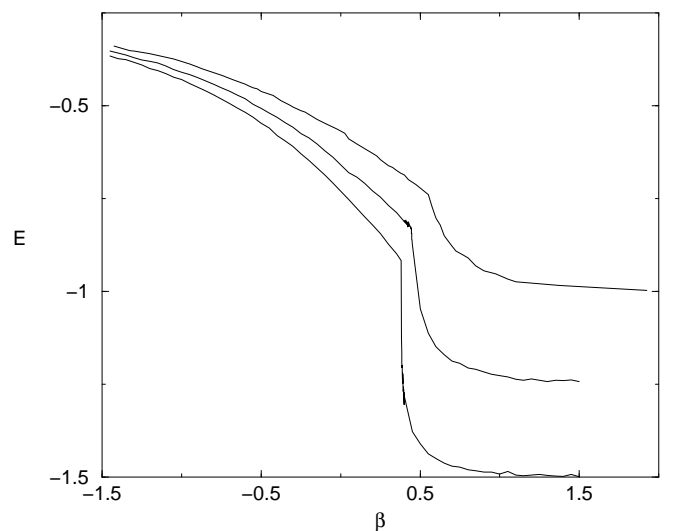

FIG. 4. The energy density $E$ as a function of the inverse temperature $\beta$ for the three connectivities $c=2,2.5,3$, from top to bottom.

Finally, in figure 5 the phase diagram of the transition to ferromagnetic blocked states is given. Below the percolation threshold of the graph $c=1$ blocked states are typically not magnetised since the graph consists of many small disconnected clusters: flipping all spins of such a cluster leads from one blocked configuration to another. As a result $\beta_{c}$ diverges as $c=1$ is approached from above. Increasing $c$ the critical value of $\beta$ and of the corresponding energy decreases monotonously as expected. Nevertheless it is interesting that the critical energy saturates already around $c \sim 4$.

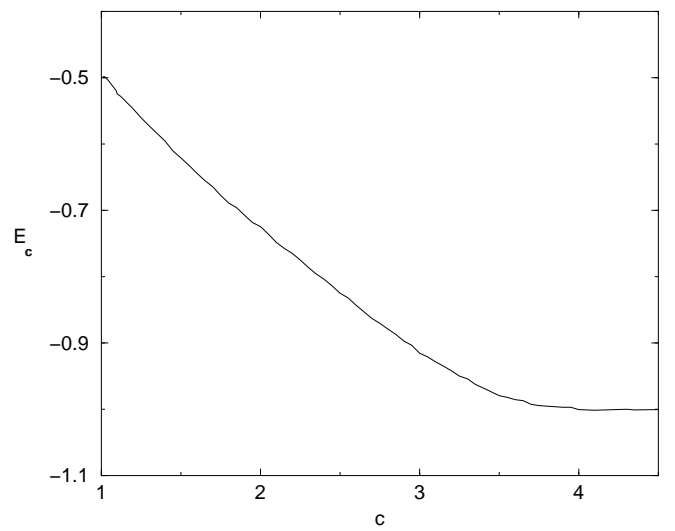

FIG. 5. Phase-diagram of the transition to ferromagnetic blocked configurations. The critical energy density $E_{c}$ is plotted against the average connectivity $c$.

\section{GENERALISATION TO THE THREE SPIN MODEL}

The generalisation to Hamiltonians with 3-spin interactions is straightforward and we only give the results. The Hamiltonian is defined by

$$
H=-\sum_{i<j<k} C_{i j k} S_{i} S_{j} S_{k}
$$

where the variable $C_{i j k}=1$ with $i<j<k$ denotes the presence of a plaquette connecting sites $i, j, k$ and $C_{i j k}=0$ denotes its absence. Choosing $C_{i j k}=1(0)$ randomly with probability $2 c / N^{2}\left(1-2 c / N^{2}\right)$ again defines an ensemble of random graphs, where each point is connected on average to $c$ plaquettes. Proceeding as in section (III) we obtain for the replicated partition function

$$
\left\langle\left\langle Z^{n}(\beta)\right\rangle\right\rangle=\prod_{\underline{\sigma}, \underline{\tau}} \int d c(\underline{\sigma}, \underline{\tau}) \exp \left\{-2 c N / 3 \sum_{\underline{\sigma}, \underline{\tau}, \underline{\rho}} c(\underline{\sigma}, \underline{\tau \rho}) c(\underline{\tau}, \underline{\rho \sigma}) c(\underline{\rho}, \underline{\sigma \tau})-c N / 3\right\}
$$




$$
\left[\sum_{\underline{\sigma}, \underline{h}} \int_{0}^{2 \pi} d \underline{\hat{h}} /(2 \pi)^{n} \exp \left\{-i \underline{h} \cdot \underline{\hat{h}}+1 / 3 \beta \underline{h} \cdot \underline{\sigma}+c \sum_{\underline{\rho}, \underline{\underline{n}}} c(\underline{\rho}, \underline{\sigma \eta}) c(\underline{\eta}, \underline{\sigma \rho}) \mathrm{e}^{i \sum_{a} \hat{h}^{a} \rho^{a} \eta^{a}}\right\} \prod_{a} \Theta\left(h^{a} \sigma^{a}\right)\right]^{N} .
$$

The self-consistent equation for $c(\underline{\sigma}, \underline{\tau})$ is

$$
c(\underline{\sigma}, \underline{\tau})=\mathrm{e}^{-\Lambda} \sum_{k=0}^{\infty} \frac{c^{k}}{k !} \prod_{l=1}^{k}\left(\sum_{\underline{\rho}_{l}, \underline{\eta}_{l}}\right) c\left(\underline{\rho_{l}}, \underline{\sigma \eta_{l}}\right) c\left(\underline{\eta_{l}}, \underline{\sigma \rho_{l}}\right) \exp \left\{1 / 3 \beta \sum_{a}\left(\tau^{a}+\sum_{l} \rho_{l}^{a} \eta_{l}^{a}\right) \sigma^{a}\right\} \prod_{a} \Theta\left(\sigma^{a}\left(\tau^{a}+\sum_{l} \rho_{l}^{a} \eta_{l}^{a}\right)\right) .
$$

Using the RS ansatz (13) and taking the limit $n \rightarrow \infty$ one obtains the self-consistent equation for $P(x, y, z)$ (14)

$$
\begin{gathered}
P(x, y, z)=\mathrm{e}^{-c} \sum_{k=0}^{\infty} \frac{c^{k}}{k !} \prod_{l=1}^{k} \int d x_{l}^{1} d y_{l}^{1} d z_{l}^{1} d x_{l}^{2} d y_{l}^{2} d z_{l}^{2} P\left(x_{l}^{1}, y_{l}^{1}, z_{l}^{1}\right) P\left(x_{l}^{2}, y_{l}^{2}, z_{l}^{2}\right) \delta\left(x-\frac{1}{4 \beta} \ln \left(\frac{f_{++} f_{+-}}{f_{-+} f_{--}}\right)\right) \\
\delta\left(y-\frac{1}{4 \beta} \ln \left(\frac{f_{++} f_{--}}{f_{-+} f_{+-}}\right)\right) \delta\left(z-\frac{1}{4 \beta} \ln \left(\frac{f_{++} f_{-+}}{f_{+-} f_{--}}\right)\right),
\end{gathered}
$$

where

$$
\begin{aligned}
f_{\sigma \tau}= & f\left(\left\{x_{l}^{1}, y_{l}^{1}, z_{l}^{1}, x_{l}^{2}, y_{l}^{2}, z_{l}^{2}\right\}, \sigma, \tau\right)=\prod_{l=1}^{k} \sum_{\rho_{l}, \eta_{l}} \exp \left\{\beta \sum_{l} x_{l}^{1} \rho_{l}+\beta \sum_{l} y_{l}^{1} \rho_{l} \eta_{l} \sigma+\beta \sum_{l} z_{l}^{1} \eta_{l} \sigma\right\} \\
& \exp \left\{\beta \sum_{l} x_{l}^{2} \eta_{l}+\beta \sum_{l} y_{l}^{2} \rho_{l} \eta_{l} \sigma+\beta \sum_{l} z_{l}^{2} \rho_{l} \sigma+\beta / 3 \sigma \sum_{l} \rho_{l} \eta_{l}+\beta / 3 \sigma \tau\right\} \Theta\left(\sigma\left(\tau+\sum_{l} \rho_{l} \eta_{l}\right)\right) .
\end{aligned}
$$

These expressions take on the same form as their counterparts in the 2-spin case (12)-(15), except that where one term $c(\underline{\sigma}, \underline{\tau})$ stood, there are now two - a simple consequence of going from bonds connecting 2 sites to plaquettes of 3 sites. For completeness we also give the free energy of metastable configurations

$$
\begin{aligned}
& \frac{1}{N}\langle\langle\ln Z\rangle\rangle=-\beta f(\beta)=-2 c / 3 \prod_{l=1}^{3} \int d x_{l} d y_{l} d z_{l} P\left(x_{l}, y_{l}, z_{l}\right) \\
& \ln \left[\frac{\sum_{\sigma, \tau, \rho} \exp \left\{\beta\left(x_{1} \sigma+y_{1} \sigma \tau \rho+z_{1} \tau \rho+x_{2} \rho+y_{2} \sigma \tau \rho+z_{2} \sigma \tau+x_{3} \tau+y_{3} \sigma \tau \rho+z_{3} \rho \sigma\right)\right\}}{\prod_{l=1}^{3}\left(2 \mathrm{e}^{\beta y_{l}} \cosh \left(\beta\left(x_{l}+z_{l}\right)\right)+2 \mathrm{e}^{-\beta y_{l}} \cosh \left(\beta\left(x_{l}-z_{l}\right)\right)\right)}\right] \\
& \quad+\mathrm{e}^{-c} \sum_{k=0}^{\infty} \frac{c^{k}}{k !} \prod_{l=1}^{k} \int d x_{l}^{1} d y_{l}^{1} d z_{l}^{1} d x_{l}^{2} d y_{l}^{2} d z_{l}^{2} P\left(x_{l}^{1}, y_{l}^{1}, z_{l}^{1}\right) P\left(x_{l}^{2}, y_{l}^{2}, z_{l}^{2}\right) \\
& \ln \left[\frac{\sum_{\sigma} \prod_{l}^{k} \sum_{\rho_{l}, \eta_{l}} \exp \left\{\beta \sum_{l}\left(x_{l}^{1} \rho_{l}+y_{l}^{1} \rho_{l} \eta_{l} \sigma+z_{l}^{1} \eta_{l} \sigma+x_{l}^{2} \eta_{l}+y_{l}^{2} \rho_{l} \eta_{l} \sigma+z_{l}^{2} \rho_{l} \sigma+1 / 3 \sigma \rho_{l} \eta_{l}\right)\right\} \Theta\left(\sigma \sum_{l} \rho_{l} \eta_{l}\right)}{\prod_{l}^{k}\left(2 \mathrm{e}^{\beta y_{l}^{1}} \cosh \left(\beta\left(x_{l}^{1}+z_{l}^{1}\right)\right)+2 \mathrm{e}^{-\beta y_{l}^{1}} \cosh \left(\beta\left(x_{l}^{1}-z_{l}^{1}\right)\right)\right)\left(2 \mathrm{e}^{\beta y_{l}^{2}} \cosh \left(\beta\left(x_{l}^{2}+z_{l}^{2}\right)\right)+2 \mathrm{e}^{-\beta y_{l}^{2}} \cosh \left(\beta\left(x_{l}^{2}-z_{l}^{2}\right)\right)\right)}\right]
\end{aligned}
$$

These equations may be solved in the same manner as described in section II , albeit with more numerical effort.

\section{MODELS WITH DISORDERED BONDS}

In this section we sketch how the formalism introduced in section II may be used to cover also models with disordered bonds. The most prominent example of this case is the two-spin model on the random graph with the signs of the bonds being \pm 1 with equal probability, the Viana-Bray model [2];

$$
H=-\sum_{i<j} J_{i j} s_{i} s_{j}
$$

The variables $J_{i j}= \pm 1$ with $i<j$ denote the presence of a bond connecting sites $i, j$ and $J_{i j}=0$ denotes its absence. For $J_{i j}= \pm 1$ with equal probability the average over the disorder in the partition function (耳) reads 


$$
\begin{aligned}
& \prod_{i<j}\left[1-c / N+c / 2 N\left(\exp \left\{i \sum_{a} \hat{h}_{i}^{a} s_{j}^{a}+i \sum_{a} \hat{h}_{j}^{a} s_{i}^{a}\right\}+\exp \left\{-i \sum_{a} \hat{h}_{i}^{a} s_{j}^{a}-i \sum_{a} \hat{h}_{j}^{a} s_{i}^{a}\right)\right\}\right] \\
& =\lim _{N \rightarrow \infty} \exp \left\{-c N / 2+c /(4 N) \sum_{i, j}\left(\mathrm{e}^{i \sum_{a} \hat{h}_{i}^{a} s_{j}^{a}+i \sum_{a} \hat{h}_{j}^{a} s_{i}^{a}}+\mathrm{e}^{-i \sum_{a} \hat{h}_{i}^{a} s_{j}^{a}-i \sum_{a} \hat{h}_{j}^{a} s_{i}^{a}}\right)\right\} .
\end{aligned}
$$

In order to decouple the two sums over the sites $i, j$ we may use the same order-parameter function $c(\underline{\sigma}, \underline{\tau})=$ $1 / N \sum_{i} \delta_{\underline{\sigma} \underline{s}_{i}} e^{\hat{h}_{i}} \cdot \underline{\tau}$ defined previously and write for (28)

$$
\exp \left\{-c N / 2+c /(4 N) \sum_{\underline{\sigma}, \underline{\tau}} c(\underline{\sigma}, \underline{\tau}) c(\underline{\tau}, \underline{\sigma})+c(\underline{\sigma}, \underline{-\tau}) c(\underline{\tau}, \underline{-\sigma})\right\} .
$$

Eliminating the conjugate order parameter we obtain $i \hat{c}(\underline{\sigma}, \underline{\tau})=c / 2(c(\underline{\tau}, \underline{\sigma})+c(-\underline{\tau},-\underline{\sigma}))$. The rest of the calculation proceeds as in the ferro-magnetic case with the order-parameter function being symmetric under the simultaneous inversion of $\underline{\sigma}$ and $\underline{\tau}$

An alternative, more cumbersome route, consists in introducing a new order-parameter function $c(\underline{\sigma}, \underline{\tau})=$ $1 / N \sum_{i} \delta_{\underline{\underline{\sigma}} \underline{s}_{i}}\left(\mathrm{e}^{\underline{\hat{h}}} \cdot \underline{\underline{\tau}}+i \mathrm{e}^{-\underline{\hat{h}}_{i} \cdot \underline{\tau}}\right)$. Using this complex global order-parameter of two arguments (28) becomes

$$
\exp \left\{-c N / 2+c /(4 N) \sum_{\underline{\sigma}, \underline{\tau}} c(\underline{\sigma}, \underline{\tau}) c^{*}(\underline{\tau}, \underline{\sigma})\right\} .
$$

The remaining calculation proceeds exactly as in the case of the ferromagnet in section II, the sole difference being the fact that $P(x, y, z)$ becomes a complex function, and equations (14)-(18) acquire complex conjugates in the appropriate places. The detailed treatment however would exceed the scope of this paper.

\section{CONCLUSION}

In this paper we considered the statistical mechanics of metastable configurations of spin models on random graphs. As a concrete example, we calculated the quenched average over the ensemble of random graphs of connectivity $c$ of the number of configurations with $h_{i} s_{i} \geq 0 \forall i$ for the case of the ferromagnetic 2-spin model. The central tool of the calculation was a global order-parameter function, which unlike the standard case of Hamiltonians composed of spin-spin interactions of various orders, takes on the form $c(\underline{\sigma}, \underline{\tau})=1 / N \sum_{i} \delta_{\underline{\sigma} \underline{s}_{i}} \mathrm{e}^{\hat{h}_{i}} \cdot \underline{\underline{\tau}}$. The replica-symmetric ansatz for such an order-parameter function of two arguments was discussed and the saddle-point equation and the free energy were derived. The saddle-point equation was solved numerically using a population-dynamics algorithm. The results were compared in detail with numerical simulations using simulated annealing and thermodynamic integration. The generalisations of this approach to 3-spin models and models with bond-disorder such as the Viana-Bray model were also discussed.

\section{ACKNOWLEDGMENTS}

Many thanks to D. Dean, S. Franz, F. Ricci-Tersenghi, and R. Zecchina for fruitful discussions.

\section{APPENDIX A:}

Here we fill in the essential steps leading to the results of section 【1]. The order-parameter function $c(\underline{\sigma}, \underline{\tau})$ is introduced via integrals over delta-functions represented by integrals over the auxiliary variables $\hat{c}(\underline{\sigma}, \underline{\tau})$. This step turns equation (8) into 


$$
\begin{gathered}
\left\langle\left\langle Z^{n}(\beta)\right\rangle\right\rangle=\prod_{\underline{\sigma}, \underline{\underline{\tau}}} \int \frac{d c(\underline{\sigma}, \underline{\tau}) d \hat{c}(\underline{\sigma}, \underline{\tau})}{2 \pi / N} \exp \left\{-i N \sum_{\underline{\sigma}, \underline{\tau}} c(\underline{\sigma}, \underline{\tau}) \hat{c}(\underline{\sigma}, \underline{\tau})-c N / 2+c N / 2 \sum_{\underline{\sigma}, \underline{\tau}} c(\underline{\sigma}, \underline{\tau}) c(\underline{\tau}, \underline{\sigma})\right\} \\
{\left[\sum_{\underline{\sigma}, \underline{\underline{h}}} \int_{0}^{2 \pi} d \underline{\hat{h}} /(2 \pi)^{n} \exp \left\{-i \underline{h} \cdot \underline{\hat{h}}+1 / 2 \beta \underline{h} \cdot \underline{\sigma}+i \sum_{\underline{\tau}} \hat{c}(\underline{\sigma}, \underline{\tau}) \mathrm{e}^{i \hat{\hat{h}} \cdot \underline{\underline{\tau}}\}} \prod_{a} \Theta\left(h^{a} \sigma^{a}\right)\right]^{N} .\right.}
\end{gathered}
$$

The auxiliary variables may be eliminated trivially by saddle-point integration giving $i \hat{c}(\underline{\sigma}, \underline{\tau})=c c(\underline{\tau}, \underline{\sigma})$. Inserting this result into (A1) gives (11). The self-consistent equation (12) follows directly from differentiating the exponent of (11) with respect to $c(\underline{\tau}, \underline{\sigma})$. The integral over $\underline{\hat{h}}$ turns into a delta-function again - so the sum over $\underline{h}$ may be performed - after the exponential term containing the order-parameter function has been expanded as a power series.

Now we need to insert the RS-ansatz (13) into the self-consistent equation (12). Collecting all terms carrying replica-indices in the $k$ th term we have

$$
\begin{aligned}
& \prod_{l}^{k} \sum_{\underline{\rho}_{l}} \exp \left\{\beta \sum_{l}\left(x_{l} \sum_{a} \rho_{l}^{a}+y_{l} \sum_{a} \rho_{l}^{a} \sigma^{a}+z_{l} \sum_{a} \sigma^{a}+1 / 2 \sum_{a} \sigma^{a} \rho_{l}^{a}\right)+\beta / 2 \sum_{a} \sigma^{a} \tau^{a}\right\} \prod_{a} \Theta\left(\sigma^{a}\left(\tau^{a}+\sum_{l} \rho_{l}^{a}\right)\right) \\
& =\prod_{a}\left(\prod_{l}^{k} \sum_{\rho_{l}^{a}} \exp \left\{\beta \sum_{l}\left(x_{l} \rho_{l}^{a}+y_{l} \rho_{l}^{a} \sigma^{a}+z_{l} \sigma^{a}+1 / 2 \sigma^{a} \rho_{l}^{a}\right)+\beta / 2 \sigma^{a} \tau^{a}\right\} \Theta\left(\sigma^{a}\left(\tau^{a}+\sum_{l} \rho_{l}^{a}\right)\right)\right) \\
& =\exp \left\{\sum_{a} \ln f_{\sigma^{a}} \tau^{a}\right\} \\
& =\exp \left\{1 / 4\left(\sum_{a} \sigma^{a}+\sum_{a} \sigma^{a} \tau^{a}+\sum_{a} \tau^{a}+n\right) \ln f_{11}+1 / 4\left(\sum_{a} \sigma^{a}-\sum_{a} \sigma^{a} \tau^{a}-\sum_{a} \tau^{a}+n\right) \ln f_{1-1}\right. \\
& \left.+1 / 4\left(-\sum_{a} \sigma^{a}-\sum_{a} \sigma^{a} \tau^{a}+\sum_{a} \tau^{a}+n\right) \ln f_{-11}+1 / 4\left(-\sum_{a} \sigma^{a}+\sum_{a} \sigma^{a} \tau^{a}-\sum_{a} \tau^{a}+n\right) \ln f_{-1-1}\right\}
\end{aligned}
$$

where $f_{\sigma \tau}$ is defined in (15). In the last step the we used

$$
\sum_{a} \delta_{\sigma \sigma^{a}} \delta_{\tau \tau^{a}}=\left\{\begin{array}{ll}
1 / 4\left(\sum_{a} \sigma^{a}+\sum_{a} \sigma^{a} \tau^{a}+\sum_{a} \tau^{a}+n\right) & \text { for } \tau=\sigma=1 \\
1 / 4\left(\sum_{a}^{a} \sigma^{a}-\sum_{a} \sigma^{a} \tau^{a}-\sum_{a} \tau^{a}+n\right) & \text { for } \tau=-1 \sigma=1 \\
1 / 4\left(-\sum_{a} \sigma^{a}-\sum_{a} \sigma^{a} \tau^{a}+\sum_{a} \tau^{a}+n\right) & \text { for } \tau=1 \sigma=-1 \\
1 / 4\left(-\sum_{a} \sigma^{a}+\sum_{a} \sigma^{a} \tau^{a}-\sum_{a} \tau^{a}+n\right) & \text { for } \tau=\sigma=-1
\end{array} .\right.
$$

Writing is $=\sum_{a} \sigma^{a}$, iu $=\sum_{a} \sigma^{a} \tau^{a}$, and $i t=\sum_{a} \tau^{a}$ the limit $n \rightarrow 0$ may be taken. Collecting all terms in $k$ and Fourier-transforming with respect to $s, u, t$ we obtain the self-consistent equation in RS in the form (14). The calculation of (16)-(18) follow the same scheme.

[1] B. Bollobas, Random Graphs (Academic Press, London, 1985)

[2] L. Viana and A. J. Bray, J. Phys C 183037 (1985)

[3] H. Orland, J. de Physique Lett.46, L763 (1995).

[4] M. Mézard and G. Parisi, J. de Physique Lett.46, L775 (1995).

[5] I. Kanter and H. Sompolinsky,Phys. Rev. Lett.58, 164 (1987).

[6] R. Monasson, S.Kirkpatrick, B.Selman, L.Troyansky, R. Zecchina, Nature 400, 133 (1999)

[7] M. Weigt and A. K. Hartmann,Phys. Rev. Lett. 84, 6118 (2000)

[8] D. De Dominicis and P. Mottishaw, J. Phys. A 20, L375 (1987),P. Mottishaw, Euro. Phys. Lett.4,333 (1987)

[9] R. Monasson, J. Phys.A 31,513 (1998)

[10] M. Mézard and G. Parisi, Eur. Phys. J. B 20, 217 (2001).

[11] S. Franz, M. Leone, F. Ricci-Tersenghi, and R. Zecchina, Phys. Rev. Lett. 87 (2001) 127209

[12] D. S Dean, Euro. Phys. J. B 15, 493 (2000), D. S. Dean and A. Lefèvre, Euro. Phys. J. B 21, 121 (2001) 
[13] J. Berg and A. Mehta, cond-mat/0108225.

[14] A. Bray and M. Moore, J. Phys. C 13, L469 (1980).

[15] S. Sastry, P.G. Debenedetti, F.H. Stillinger, Nature 393, 554 (1998)

[16] G. Biroli and R. Monasson Europhys. Lett. 50, 155 (2000).

[17] A. Barrat, J. Kurchan,V. Loreto, and M. Sellitto, Phys. Rev. Lett.85,5034 (2000); A. Barrat, J. Kurchan, V. Loreto and M. Sellitto, Phys. Rev.E 63, 051301 (2001)

[18] J. Berg and A. Mehta, to appear in Europhys. Lett., cond-mat/0012416

[19] D. S. Dean and A. Lefèvre, Phys. Rev. Lett. 86, 5639 (2001), A. Lefèvre and D. S. Dean, J. Phys.A34 L213 (2001), D. S. Dean and A. Lefèvre, cond-mat/0106220.

[20] G. Biroli and M. Mézard, cond-mat/0106309

[21] A. Barrat and R. Zecchina, Phys. Rev. E 59 R1299 (1999).

[22] M. Mézard,G. Parisi, and M. Virasoro, Spin glass theory and beyond, World Scientific, Singapore (1987).

[23] For a recent application of this method see: H. Makse and J. Kurchan, cond-mat/0107163. 